\title{
Metodologia para determinação das necessidades nutricionais de melão e melancia ${ }^{1}$
}

\author{
José A. de A. Paula ${ }^{2}$, José $F$. de Medeiros $^{2}$, Neyton de 0 . Miranda ${ }^{2}$, \\ Francisco de A. de O liveira $^{2} \&$ Carlos J. G. de S. Lima ${ }^{2}$
}

\begin{abstract}
RESU M O
Realizou-se este trabalho visando definir a necessidade nutricional de nitrogênio, fósforo e potássio de melão e melancia, em função da produtividade esperada, a partir de dados médios citados na literatura, e validá-los com dados obtidos em experimentos com melão e melancia, realizados no Agropolo Assu/ Mossoró. 0 experimento com melão seguiu o delineamento em blocos ao acaso, com três repetições, no esquema fatorial $3 \times 3+3$, que constou da combinação de três doses de $\mathrm{N}\left(\mathrm{N}_{1}=42, \mathrm{~N}_{2}=84\right.$ e $\left.\mathrm{N}_{3}=126 \mathrm{~kg} \mathrm{ha}^{-1}\right)$ e de $\mathrm{K}\left(\mathrm{K}_{1}=106, \mathrm{~K}_{2}=212\right.$ e K $\left.3=322 \mathrm{~kg} \mathrm{ha}^{-1}\right)$, mais três tratamentos adicionais $\left(\mathrm{N}_{0} \mathrm{~K}_{0^{\prime}}\right.$ $\mathrm{N}_{0} \mathrm{~K}_{2}$ e $\mathrm{N}_{2} \mathrm{~K}_{0}$ ), sendo que $\mathrm{N}_{0}$ e $\mathrm{K}_{0}$ não receberam N nem $\mathrm{K}$. $\mathrm{O}$ experimento com melancia seguiu o mesmo delineamento experimental, modificando as doses de $\left.\mathrm{N}\left(\mathrm{N}_{1}=45, \mathrm{~N}_{2}=91 \text { e } \mathrm{N}_{3}=136 \mathrm{~kg} \mathrm{ha}\right)^{-1}\right)$ e de $\mathrm{K}$ $\left(\mathrm{K}_{1}=78, \mathrm{~K}_{2}=156\right.$ e $\left.\mathrm{K}_{3}=234 \mathrm{~kg} \mathrm{ha}^{-1}\right)$, havendo dois tratamentos adicionais $\left(\mathrm{N}_{0} \mathrm{~K}_{2}^{3}\right.$ e $\left.\mathrm{N}_{2} \mathrm{~K}_{0}\right)$. O modelo que melhor se ajustou, tanto para a melancia como para o melão, seguiu a relação produtividade ( $\mathrm{kg}$ planta ${ }^{-1}$ ) $x$ nutrientes exportados (g planta-1 ${ }^{-1}$. O s índices de concordância observados entre os valores estimados pelo modelo de absorção total de $\mathrm{N}$ e K e valores obtidos experimentalmente, são considerados aceitáveis, tanto para melancia como para melão.
\end{abstract}

Palavras-chave: Citrullus lanatus, Cucumis melo, absorção de nutrientes, modelagem

\section{M etodology for assessment of nutritional requirements of melon and watermelon}

\begin{abstract}
This work was carried out aiming to determine nutritional requirements of nitrogen, phosphorus and potassium for melon and watermelon, as a function of estimated yield, and validate them $w$ ith experimental data obtained from melon and watermelon trials carried out in Assu-M ossoró region. M elon trial follow ed a randomized block design, in a $3 \times 3+3$ factorial scheme with three replications, which consisted of combination of three doses of $\mathrm{N}\left(\mathrm{N}_{1}=42, \mathrm{~N}_{2}=84\right.$ and $\left.\mathrm{N}_{3}=126 \mathrm{~kg} \mathrm{ha}^{-1}\right)$ and of $\mathrm{K}\left(\mathrm{K}_{1}=106, \mathrm{~K}_{2}=212\right.$ and $\left.\mathrm{K}_{3}=322 \mathrm{~kg} \mathrm{ha}^{-1}\right)$, and three additional treatments $\left(\mathrm{N}_{0} \mathrm{~K}_{0}, \mathrm{~N}_{0} \mathrm{~K}_{2}\right.$ e $\left.\mathrm{N}_{2} \mathrm{~K}_{0}\right)$, where $\mathrm{N}_{0}$ and $\mathrm{K}_{0}$ had no application of $\mathrm{N}$ and $\mathrm{K}$. Watermelon trial followed the same design, with modification of doses of $\mathrm{N}$ $\left(\mathrm{N}_{1}=45, \mathrm{~N}_{2}=91\right.$ and $\left.\mathrm{N}_{3}=136 \mathrm{~kg} \mathrm{ha}^{-1}\right)$ and $\mathrm{K}\left(\mathrm{K}_{1}=78, \mathrm{~K}_{2}=156\right.$ and $\left.\mathrm{K}_{3}=234 \mathrm{~kg} \mathrm{ha}^{-1}\right)$, with two additional treatments $\left(\mathrm{N}_{0} \mathrm{~K}_{2}\right.$ and $\left.\mathrm{N}_{2} \mathrm{~K}_{0}\right)$. The model of better adjust, both for melon and watermelon, followed the relation of yield $\left(\mathrm{kg}\right.$ planta $\left.{ }^{-1}\right) \times$ exported nutrients $\left(\mathrm{g}\right.$ planta-1 ${ }^{-1}$. Agreement indexes observed between estimated values of total absorption of $\mathrm{N}$ and $\mathrm{K}$ and values obtained experimentally were considered acceptable, for melon and watermelon.
\end{abstract}

Key words: Citrullus Ianatus, Cucumis melo, nutrient absorption, modeling 


\section{INTRODUÇÃO}

O melão e a melancia são culturas importantes na pauta de exportações do estado do Rio Grande do Norte, sobretudo a região do Agropolo Mossoró/Assu. Segundo Gurgel et al. (2010), isto ocorre devido às condições edafoclimáticas (solo, temperatura, umidade relativa do ar, etc.) e à disponibilidade de mananciais de água superficial e subterrânea, de maneira que as culturas podem ser conduzidas totalmente sob irrigação e receber nutrientes via fertirrigação.

A absorção de grandes quantidades de nutrientes em curtos períodos de tempo caracteriza a suma exigência nutricional das hortaliças, entre as quais as folhosas e tuberosas, que deixam poucos restos culturais no solo e consideradas altamente esgotantes. Neste aspecto fica evidente a necessidade de se conhecer o balanço de nutrientes de cada cultura para manejar a adubação, escolher culturas para rotação e otimizar a utilização de insumos.

Uma informação relevante para a recomendação de adubação é a proporção adequada entre os nutrientes N, P e K, a qual, em uma adubação completa, deve ser 1:1,5:1,7; a importância do balanceamento entre nutrientes é ressaltada por Kanwar \& Sekhon (1998). A partir desta informação as recomendações devem englobar as exigências nutricionais de cada cultura, a capacidade de fornecimento de nutrientes pelo solo, a eficiência de absorção de nutrientes e a produtividade esperada, além de serem baseadas, sempre, em dados ajustados às condições locais de plantio. Outra observação significativa é o fato de que a fertirrigação é mais eficiente quando a acidez e os teores de nutrientes do solo são corrigidos anteriormente para valores considerados adequados ao bom desenvolvimento da cultura explorada.

As quantidades de nutrientes requeridas pelas culturas podem ser estimadas por diferentes métodos, um dos quais recomendado por Santos et al. (2008), inclusive programas computacionais como o FERTICALC ${ }^{\circledR}$ (Novais \& Smyth, 1999). Em recomendações para bananeira, o programa produziu recomendações nas quais as doses de nutrientes aumentaram continuamente com o aumento da produtividade esperada e com a diminuição dos teores desses nutrientes no solo, sendo maiores no primeiro ciclo e menores a partir do segundo ciclo (Oliveira et al., 2005).

Entre várias formas de parcelamento da aplicação de $\mathrm{N}, \mathrm{P}_{2} \mathrm{O}_{5}$ e $\mathrm{K}_{2} \mathrm{O}$ para a cultura do melão (Faria et al., 1994; Pinto et al., 1993; Soares et al., 1999), a recomendação é, respectivamente, de 10, 80 e 10\%, em fundação, e 90, 20 e $90 \%$ em cobertura; a de Montag (1999), em fundação, é de 15 a $25 \%$ de N, $100 \%$ de $\mathrm{P}_{2} \mathrm{O}_{5}$ e 20 a $30 \%$ de $\mathrm{K}_{2} \mathrm{O}$ e de 75 a $85 \%$, $0 \%$ e 70 a $80 \%$, respectivamente, em cobertura. A recomendação de Novais \& Smyth (1999), semelhante à de Papadopoulos (1999), é de 35 a $50 \%$ de N, $100 \%$ de $\mathrm{P}_{2} \mathrm{O}_{5}$ e 60 a $100 \%$ de $\mathrm{K}_{2} \mathrm{O}$ em fundação e 50 a $65 \%, 0 \%$ e 0 a $40 \%$ em cobertura, respectivamente.

A redução nas perdas de nutrientes por lixiviação, principalmente do nitrogênio, em virtude do parcelamento das adubações, é ressaltada por diversos autores. Além disso e segundo Gärdenäs et al. (2005), a distribuição do nitrogênio é mais uniforme no perfil do solo quando se utiliza fertirrigação por gotejamento, havendo uma distribuição melhor do nutriente no bulbo úmido e zona radicular, exceto por uma pequena concentração ao redor da frente de molhamento.

A adoção da fertirrigação tem, como consequência, a dificuldade em determinar as dosagens adequadas de nutrientes a serem aplicadas às culturas para que elas atinjam a máxima eficiência da absorção (Holanda Filho et al., 2006). Neste sentido, existem sugestões de fatores de correção para as quantidades totais de N, P e K exportados pela planta. Os fatores sugeridos por Montag (1999) são 1,2 a 1,5 para N, 1,9 a 2,2 para P e 1,4 a 1,6 para $\mathrm{K}$, na adubação convencional, e 1,1 a 1,2; 1,6 a 1,9 e 1,2 a 1,4 , respectivamente, na fertirrigação.

Em estudo referente ao efeito da adubação com $\mathrm{N}$ e K na produção de melão em solos arenosos, a produtividade da cultura aumentou com o aumento dos níveis de $\mathrm{N}$, até uma produtividade máxima com $120 \mathrm{~kg} \mathrm{ha}^{-1}$ de $\mathrm{N}$ aplicado via água de irrigação, a qual não diferiu estatisticamente de $180 \mathrm{~kg} \mathrm{ha}^{-1}$ de $\mathrm{N}$, que elevou a produtividade em apenas 7,8\% (Coelho et al., 2001).

Estudo semelhante realizado em Latossolo Vermelho-amarelo Distrófico típico de Piracicaba, SP, Kano et al. (2010) avaliaram a extração de nutrientes pelo meloeiro rendilhado híbrido Bônus $\mathrm{n}^{\circ} 2$ com ciclo total de 86 dias, adubado via água de irrigação por gotejamento em ambiente protegido. A produtividade total por planta foi de $1,80 \mathrm{~kg}$, obtida com a aplicação de $200 \mathrm{~kg} \mathrm{ha}^{-1} \mathrm{de}$ $\mathrm{N}$, na forma de Nitrato de Amônio até os 64 dias após o transplante e na forma de Nitrato de Potássio, até 72 dias. A dose de $340 \mathrm{~kg} \mathrm{ha}^{-1} \mathrm{de} \mathrm{K}_{2} 0$ foi aplicada na forma de Nitrato de Potássio, até 72 dias após o transplante.

Objetivou-se com esse trabalho foi avaliar uma metodologia para definir a necessidade nutricional de nitrogênio, fósforo e potássio, nas culturas estudadas.

\section{Material E MÉTODOS}

O modelo avaliado seguiu recomendação de Vivancos (1999) e relaciona as quantidades totais de $\mathrm{N}, \mathrm{P}$ e K acumulados pela planta na produtividade esperada (total exportado $=\mathrm{f}$ (produtividade)), segundo curva polinomial do segundo grau. Para verificar sua eficiência, o modelo foi comparado com outros, tais como quantidade de nutriente $\left(\mathrm{g}_{\text {planta }}{ }^{-1}\right)=\mathrm{f}$ (produtividade $\left.\left(\mathrm{Mg} \mathrm{ha}^{-1}\right)\right)$ e quantidade de Nutriente $\left(\mathrm{g}\right.$ planta $\left.{ }^{-1}\right)$ $=\mathrm{f}\left(\right.$ produtividade $\left.\left(\mathrm{kg} \mathrm{planta}^{-1}\right)\right)$.

O melhores modelos citados foram confrontados com o modelo da derivada primeira do polinômio do segundo grau, proposto por Vivancos (1999), que relaciona Quantidade do Nutriente $\left(\mathrm{kg} \mathrm{Mg}^{-1}\right)=\mathrm{f}\left(\right.$ produtividade $\left.\left(\mathrm{Mg} \mathrm{ha}^{-1}\right)\right)$, além do modelo que relaciona Quantidade do Nutriente $\left(\mathrm{kg} \mathrm{Mg}^{-1}\right)=\mathrm{f}$ (produtividade ( $\left.\mathrm{kg} \mathrm{planta}^{-1}\right)$ ) para polinômio do primeiro grau.

Lançou-se mão, para determinar as equações de regressão dos nutrientes $\mathrm{N}-\mathrm{P}-\mathrm{K}$ para melancia, dos dados totais de nutrientes acumulados na planta obtidos por Grangeiro \& CecílioFilho (2003; 2004), Grangeiro et al. (2005) e Serafim(2006); para melão, utilizaram-se dados de Lima (2001), Vivancos (1999), Medeiros et al. (2008), Temóteo (2010), Silva Júnior (2006) e Bar-Yosef (1999).

A determinação dos dados e a obtenção das curvas de produtividade versus quantidade do nutriente $\left(\mathrm{g}\right.$ planta $^{-1}$ ou 
$\mathrm{kg} \mathrm{Mg}^{-1}$ ) para cada cultura, foram realizadas de acordo com o procedimento adotado por Paula (2007). Visando à transformação das quantidades líquidas de nutrientes obtidos pelas curvas "produtividade versus quantidade do nutriente (g planta-1 ou $\mathrm{kg} \mathrm{Mg}^{-1}$ )" para $\mathrm{kg} \mathrm{ha}^{-1}$, adotou-se o seguinte procedimento: para a determinação das quantidades semanal ou diária de nutrientes aplicadas na adubação e/ou fertirrigação do melão e da melancia, utilizou-se uma curva média obtida entre variedades que deram origem às equações das curvas "total exportado" vs. "produtividade média", utilizaram-se períodos igualmente espaçados nas variedades para as quais se determinaram as equações que calibram os totais exportados em N, P e K, das respectivas culturas em relação à produtividade almejada, conforme recomendações propostas por Montag (1999).

Para avaliar a eficiência da metodologia assumiram-se dados médios comuns aos das áreas cultivadas com melão e melancia analisados pelo Laboratório de Solos da Universidade Federal Rural do Semiárido - UFERSA, acrescidos aos dados das áreas dos experimentos com melão e com melancia, totalizando oito exemplos para o nutriente $\mathrm{K}\left(\mathrm{K}_{1}, \mathrm{~K}_{2}, \mathrm{~K}_{3}, \mathrm{~K}_{4}, \mathrm{~K}_{5}, \mathrm{~K}_{6}, \mathrm{~K}_{7}, \mathrm{~K}_{8}\right)$, dois exemplos para o nutriente $\mathrm{P}\left(\mathrm{P}_{1}\right.$ e $\left.\mathrm{P}_{2}\right)$ e um exemplo para o nutriente $\mathrm{N}\left(\mathrm{N}_{1}\right)$ para cada cultura. Adotaram-se, ainda, 36 e 28 $\mathrm{Mg} \mathrm{ha}^{-1}$ como produtividade almejada para melão e melancia, respectivamente.

Para o experimento com melão, realizado com a cultivar tipo Gália 'Solar Net' em espaçamento de 1,85 x 0,40, utilizou-se o delineamento em blocos casualizados arranjados no esquema fatorial $3 \times 3+3$, com três repetições, em que cada parcela correspondeu a uma fileira de $9 \mathrm{~m}$. Os tratamentos constaram de combinações das doses de $\mathrm{N}\left(\mathrm{N}_{1}=42, \mathrm{~N}_{2}=84\right.$ e $\mathrm{N}_{3}=126 \mathrm{~kg}$ $\left.\mathrm{ha}^{-1}\right)$ e de $\mathrm{K}_{2} \mathrm{O}\left(\mathrm{K}_{1}=106, \mathrm{~K}_{2}=212 \mathrm{e} \mathrm{K}_{3}=322 \mathrm{~kg} \mathrm{ha}^{-1}\right)$ aplicados via fertirrigação, mais três tratamentos adicionais $\left(\mathrm{N}_{0} \mathrm{~K}_{0}, \mathrm{~N}_{0} \mathrm{~K}_{2}\right.$ e $\mathrm{N}_{2} \mathrm{~K}_{0}$, com $\mathrm{N}_{0}$ e $\mathrm{K}_{0}$ denotando doses zero). A adubação de fundação constou da aplicação de $108 \mathrm{~kg} \cdot \mathrm{ha}^{-1}$ de superfosfato simples e $162 \mathrm{~kg} \mathrm{ha}^{-1}$ de monoamônico fosfato (MAP, 10-5200), totalizando $102 \mathrm{~kg} \mathrm{ha}^{-1} \mathrm{de}_{2} \mathrm{O}_{5}$. O complemento nutricional do fósforo foi realizado via fertirrigação, utilizando-se ácido fosfórico, totalizando $70 \mathrm{~kg} \mathrm{ha}^{-1}$ de $\mathrm{P}_{2} \mathrm{O}_{5}$.

Para o experimento com melancia, realizado com a cultivar Mickylee, em espaçamento de 2,0 x 0,50, adotou-se o delineamento experimental em blocos casualizados em esquema fatorial $3 \times 3+2$, com três repetições, sendo cada parcela experimental constituída de três fileiras de $12,0 \mathrm{~m}$ de comprimento. A semeadura foi realizada em bandejas de poliestireno preenchidas com substrato comercial Goldmix $47^{\circ}$. Os tratamentos resultaram da combinação de três doses de nitrogênio $\left(\mathrm{N}_{1}=45, \mathrm{~N}_{2}=91\right.$ e $\left.\mathrm{N}_{3}=136 \mathrm{~kg} \mathrm{ha}^{-1}\right)$ e três doses de potássio $\left(\mathrm{K}_{1}=78, \mathrm{~K}_{2}=156 \mathrm{e} \mathrm{K}_{3}=234 \mathrm{~kg} \mathrm{ha}^{-1}\right)$, além de dois tratamentos adicionais: $\left(\mathrm{N}_{0} \mathrm{~K}_{2} \mathrm{e} \mathrm{N}_{2} \mathrm{~K}_{0}\right)$, sendo $\mathrm{N}_{2}$ e $\mathrm{K}_{2}$ a dose recomendada para fertilização e $\mathrm{N}_{0} \mathrm{eK}_{0}$ significando dose zero. A adubação de fundação constou da aplicação do composto orgânico Poli Fértil ${ }^{\circledR}$ na quantidade de $4 \mathrm{t} \mathrm{ha}^{-1}$ e da adubação fosfatada com $50 \mathrm{~kg} \mathrm{ha}^{-1}$ na forma de superfosfato simples. $\mathrm{Na}$ fertirrigação foram utilizados uréia, ácido nítrico, nitrato de cálcio, cloreto de potássio e sulfato de potássio.

Realizada a colheita dos frutos (63 dias após semeadura), no mesmo local foram realizadas as determinações de produtividade, que constaram da análise do peso médio do fruto, número de frutos por planta e produção total.

Para os nutrientes absorvidos pelas culturas, as análises laboratoriais foram realizadas no Laboratório de Solos do Departamento de Ciências Ambientais da Universidade Federal Rural do Semiárido (UFERSA), atentando-se para o seguinte procedimento: para determinação da massa seca as amostras foram mantidas em estufa de circulação forçada com temperatura regulada para $65-70{ }^{\circ} \mathrm{C}$, até que se obtivesse peso constante (três a quatro dias) em balança eletrônica com precisão de 0,01 $\mathrm{g}$; na determinação dos teores dos nutrientes utilizou-se $0,2 \mathrm{~g}$ da massa seca que foi digerida utilizando-se ácido sulfúrico, peróxido de hidrogênio, sulfatos de sódio e de cobre e selênio (Tedesco et al., 1995); quantificou-se o nitrogênio total através da destilação por arrasto de vapores, o fósforo por espectrofotometria e o potássio por fotometria de emissão de chama, conforme recomendado por Silva (1999). Os dados foram submetidos às análises de variância e de regressão para o modelo polinomial completo contido no software SAEG, versão 8.0 (Ribeiro Júnior, 2001) a pelo menos 5\% de probabilidade.

Para confirmação da eficiência do sistema, os dados obtidos a partir da simulação foram confrontados com os resultados dos experimentos de campo realizados com o objetivo de se estudar doses de $\mathrm{N}$ e K aplicados na adubação/fertirrigação na produção das plantas de melão e da melancia.

O teste utilizado para medir a eficiência foi o Índice de Concordância "d" como utilizado por Teixeira et al. (2005) e por Pereira et al. (2009). Utilizaram-se, no teste, somente os tratamentos dos experimentos de campo comuns às duas culturas, ou seja, $\mathrm{N}_{1} \mathrm{~K}_{1}, \mathrm{~N}_{2} \mathrm{~K}_{2}, \mathrm{~N}_{3} \mathrm{~K}_{3}, \mathrm{~N}_{2} \mathrm{~K}_{0}$ e $\mathrm{N}_{0} \mathrm{~K}_{2}$.

\section{RESULTADOS E DISCUSSÃO}

Obtiveram-se, para ambas as culturas, as equações determinadas apenas com os dados de produtividades totais, já que se constatou baixa quantidade de dados na literatura, apresentando informações com produtividade comercial. Além disso, tentativas em se obter tais equações com os poucos dados existentes (dados de produtividades comerciais do melão), resultaram em baixos coeficientes de determinação $\left(R^{2}\right)$.

Para a melancia foram obtidos, no ajustamento das equações, resultados satisfatórios com a utilização da totalidade dos dados coletados na literatura (oito dados). Para o melão não foi possível a obtenção das curvas com a totalidade dos dados coletados (onze dados), já que no trabalho de Temóteo (2010) não havia dados de fósforo e não foi possível se obter dados de Vivancos (1999) quando se relacionaram totais exportados $\left(\mathrm{g}_{\text {planta }}{ }^{-1}\right)=\mathrm{f}\left(\right.$ produtividade $\left.\left(\mathrm{kg} \mathrm{planta}^{-1}\right)\right)$, sendo então eliminados dois dados de $\mathrm{N}$, cinco de $\mathrm{P}$ e dois de $\mathrm{K}$ resultando, para esses nutrientes, 9, 6 e 9 dados, respectivamente (Tabelas 1 a 3$)$.

Dos diferentes modelos testados (Tabelas 1 a 3 ) e se considerando os três nutrientes estudados (N, P e K) e as culturas do melão e da melancia, os melhores foram aqueles que relacionaram totais exportados $\left(\mathrm{g}_{\text {planta }}{ }^{-1}\right)=$ $\mathrm{f}$ (produtividade, $\mathrm{kg}_{\text {planta-1 }}$ ) através de polinômio do $1^{\mathrm{o}}$ grau. 
Tabela 1. Equações* das curvas de calibração dos Totais exportados de $N$ versus produtividade da melancia e do melão para o modelo de nutrição de N

\begin{tabular}{|c|c|c|c|c|}
\hline Cultura & Relação & Número de dados & Equações do total exportado de $\mathrm{N}$ & $\mathbf{R}^{2}$ \\
\hline Melão & $\begin{array}{l}\left.\text { (g planta }{ }^{-1}\right) \times\left(\mathrm{kg} \mathrm{planta}^{-1}\right) \\
\left(\mathrm{g} \mathrm{planta}^{-1}\right) \times\left(\mathrm{kg} \mathrm{planta}^{-1}\right) \\
\left(\mathrm{g} \mathrm{planta}^{-1}\right) \times\left(\mathrm{Mg} \mathrm{ha}^{-1}\right) \\
\left(\mathrm{g} \mathrm{planta}^{-1}\right) \times\left(\mathrm{Mg} \mathrm{ha}^{-1}\right) \\
\left(\mathrm{kg} \mathrm{Mg}^{-1}\right) \times\left(\mathrm{kg} \mathrm{planta}^{-1}\right) \\
\left(\mathrm{kg} \mathrm{Mg}^{-1}\right) \times\left(\mathrm{Mg} \mathrm{ha}^{-1}\right)\end{array}$ & $\begin{array}{l}9 * * \\
9 \\
9 \\
9 \\
9 \\
9\end{array}$ & $\begin{array}{l}y=5,8952 x-8,2243 \\
y=-0,5756 x^{2}+10,625 x-16,627 \\
y=0,399 x-3,9601 \\
y=-0,0007 x^{2}+0,5158 x-6,7481 \\
y=0,638 x+0,9675 \\
y=0,0726 x+0,6758\end{array}$ & $\begin{array}{l}0,9673 \\
0,9766 \\
0,4543 \\
0,5354 \\
0,7006 \\
0,7429\end{array}$ \\
\hline Melancia & $\begin{array}{l}\left.\text { (g planta } \mathrm{C}^{-1}\right) \times\left(\mathrm{kg} \mathrm{planta}^{-1}\right) \\
(\mathrm{g} \text { planta } \\
(\mathrm{g} \text { planta } \\
-1) \times\left(\mathrm{kg} \mathrm{planta}^{-1}\right) \times\left(\mathrm{Mg} \mathrm{ha}^{-1}\right) \\
(\mathrm{g} \text { planta } \\
\left(\mathrm{kg} \mathrm{Mg}^{-1}\right) \times\left(\mathrm{Mg} \mathrm{ha}^{-1}\right) \\
\left(\mathrm{kg} \mathrm{Mg} \mathrm{planta}^{-1}\right) \times\left(\mathrm{Mg} \mathrm{ha}^{-1}\right)\end{array}$ & $\begin{array}{l}8^{* *} \\
8 \\
8 \\
8 \\
8 \\
8\end{array}$ & $\begin{array}{l}y=1,202 x+0,8979 \\
y=-0,1695 x^{2}+4,8081 x-14,323 \\
y=-0,185 x+19,978 \\
y=-0,0136 x^{2}+0,9863 x-2,0403 \\
y=0,011 x+1,1386 \\
y=0,0032 x+1,2301\end{array}$ & $\begin{array}{l}0,7213 \\
0,8713 \\
0,1666 \\
0,2677 \\
0,0154 \\
0,0169\end{array}$ \\
\hline
\end{tabular}

* y representa os valores de cada nutriente e $\mathrm{x}$ os valores de produtividade

** curva adotada para validação

Tabela 2. Equações* das curvas de calibração dos Totais exportados $x$ produtividade da melancia e do melão para 0 modelo de nutrição de $P$

\begin{tabular}{|c|c|c|c|c|}
\hline Cultura & Relação & Número de dados & Equações do total exportado de $\mathbf{P}$ & $\mathbf{R}^{2}$ \\
\hline Melão & 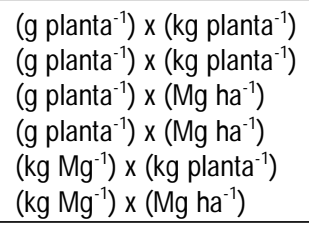 & $\begin{array}{l}6^{* *} \\
6 \\
6 \\
6 \\
6 \\
6 \\
\end{array}$ & $\begin{array}{l}y=1,235 x-1,9479 \\
y=0,1088 x^{2}+0,3334 x-0,2998 \\
y=0,0843 x-0,64 \\
y=0,0007 x^{2}+0,0214 x+0,5836 \\
y=0,1273 x+0,1747 \\
y=0,0064 x+0,437\end{array}$ & $\begin{array}{l}0,9397 \\
0,9490 \\
0,4877 \\
0,4904 \\
0,7340 \\
0,2205\end{array}$ \\
\hline Melancia & $\begin{array}{l}\left.\text { (g planta }{ }^{-1}\right) \times\left(\mathrm{kg} \mathrm{planta}^{-1}\right) \\
\left(\mathrm{g} \mathrm{planta}^{-1}\right) \times\left(\mathrm{kg} \mathrm{planta}^{-1}\right) \\
\left(\mathrm{g} \mathrm{planta}^{-1}\right) \times\left(\mathrm{Mg} \mathrm{ha}^{-1}\right) \\
\left(\mathrm{g} \mathrm{planta}^{-1}\right) \times\left(\mathrm{Mg} \mathrm{ha}^{-1}\right) \\
\left(\mathrm{kg} \mathrm{Mg}^{-1}\right) \times\left(\mathrm{kg} \mathrm{planta}^{-1}\right) \\
\left(\mathrm{kg} \mathrm{Mg}^{-1}\right) \times\left(\mathrm{Mg} \mathrm{ha}^{-1}\right)\end{array}$ & $\begin{array}{l}8^{* *} \\
8 \\
8 \\
8 \\
8 \\
8\end{array}$ & $\begin{array}{l}y=0,0475 x+0,508 \\
y=-0,001 x^{2}+0,0686 x+0,4187 \\
y=-0,0136 x+1,5219 \\
y=-0,0003 x^{2}+0,0118 x+1,0455 \\
y=-0,0076 x+0,1947 \\
y=-0,0002 x+0,1305\end{array}$ & $\begin{array}{l}0,6785 \\
0,6816 \\
0,7073 \\
0,7446 \\
0,6186 \\
0,0065\end{array}$ \\
\hline
\end{tabular}

* y representa os valores de cada nutriente e $\mathrm{x}$ os valores de produtividade

** curva adotada para validação

Tabela 3. Equações* das curvas de calibração dos Totais exportados $x$ produtividade da melancia e do melão para 0 modelo de nutrição de $\mathrm{K}$

\begin{tabular}{cllll}
\hline Cultura & \multicolumn{1}{c}{ Relação } & Número de dados & Equações do total exportado de $\mathbf{K}$ & $\mathbf{R}^{2}$ \\
Melão & $\left(\right.$ g planta $\left.^{-1}\right) \times\left(\mathrm{kg} \mathrm{planta}^{-1}\right)$ & $9 * *$ & $\mathrm{y}=4,5784 \mathrm{x}+2,6016$ & 0,7085 \\
& $\left(\mathrm{~g} \mathrm{planta}^{-1}\right) \times\left(\mathrm{kg} \mathrm{planta}^{-1}\right)$ & 9 & $\mathrm{y}=0,835 \mathrm{x}^{2}-2,2827 \mathrm{x}+14,79$ & 0,7322 \\
& $\left(\mathrm{~g} \mathrm{planta}^{-1}\right) \times\left(\mathrm{Mg} \mathrm{ha}^{-1}\right)$ & 9 & $\mathrm{y}=0,3283 \mathrm{x}+5,7113$ & 0,3316 \\
& $\left(\mathrm{~g} \mathrm{planta}^{-1}\right) \times\left(\mathrm{Mg} \mathrm{ha}^{-1}\right)$ & 9 & $\mathrm{y}=0,0055 \mathrm{x}^{2}-0,0064 \mathrm{x}+8,4609$ & 0,5486 \\
& $\left(\mathrm{~kg} \mathrm{Mg}^{-1}\right) \times\left(\mathrm{kg} \mathrm{planta}^{-1}\right)$ & 9 & $\mathrm{y}=-0,266 \mathrm{x}+6,3939$ & 0,0698 \\
& $\left(\mathrm{~kg} \mathrm{Mg}^{-1}\right) \times\left(\mathrm{Mg} \mathrm{ha}^{-1}\right)$ & 9 & $\mathrm{y}=0,0556 \mathrm{x}+3,235$ & 0,2360 \\
\hline Melancia & $\left(\right.$ g planta $\left.^{-1}\right) \times\left(\mathrm{kg} \mathrm{planta}^{-1}\right)$ & $8 * *$ & $\mathrm{y}=1,934 \mathrm{x}+3,0964$ & 0,7180 \\
& $\left(\mathrm{~g} \mathrm{planta}^{-1}\right) \times\left(\mathrm{kg} \mathrm{planta}^{-1}\right)$ & 8 & $\mathrm{y}=-0,193 \mathrm{x}^{2}+6,0394 \mathrm{x}-14,232$ & 0,7927 \\
& $\left(\mathrm{~g} \mathrm{planta}^{-1}\right) \times\left(\mathrm{Mg} \mathrm{ha}^{-1}\right)$ & 8 & $\mathrm{y}=-0,0919 \mathrm{x}+24,941$ & 0,0173 \\
& $\left(\mathrm{~g} \mathrm{planta}^{-1}\right) \times\left(\mathrm{Mg} \mathrm{ha}^{-1}\right)$ & 8 & $\mathrm{y}=-0,019 \mathrm{x}^{2}+1,5377 \mathrm{x}-5,6939$ & 0,0993 \\
& $\left(\mathrm{~kg} \mathrm{Mg}^{-1}\right) \times\left(\mathrm{kg} \mathrm{planta}^{-1}\right)$ & 8 & $\mathrm{y}=0,0126 \mathrm{x}+2,0288$ & 0,0031 \\
& $\left(\mathrm{~kg} \mathrm{Mg}^{-1}\right) \times\left(\mathrm{Mg} \mathrm{ha}^{-1}\right)$ & 8 & $\mathrm{y}=0,0372 \mathrm{x}+0,8462$ & 0,3890 \\
\hline
\end{tabular}

* y representa os valores de cada nutriente e $\mathrm{x}$ os valores de produtividade

** curva adotada para validação

A melhor relação obtida para $\left(\mathrm{g}\right.$ planta $\left.{ }^{-1}\right)$ versus $\left(\mathrm{kg} \mathrm{planta}^{-1}\right)$ demonstra que, para as culturas estudadas, há interferência da quantidade de nutrientes aplicados para diferentes espaçamentos, considerados na linha e entre linhas das plantas. Além disso, não houve diferenças estatísticas para os coeficientes de determinação da reta $\left(R^{2}\right)$ entre as equações polinomiais do primeiro e segundo graus no intervalo de produtividade estudado, caso em que se adotou a relação polinomial do $1^{\circ}$ grau pela maior simplicidade, discordando de Vivancos (1999) que afirma haver uma relação polinomial do segundo grau entre os parâmetros estudados.

De maneira geral pode-se afirmar que as equações que usam os totais exportados em função da produtividade almejada para melancia e melão, para o modelo proposto, evidenciaram altos coeficientes de determinação, parâmetro também usado por Monteiro et al. (2006), para avaliar modelo de resposta do meloeiro à fertirrigação.

A produtividade do experimento com melão variou de 18,44 $\left(\mathrm{N}_{0} \mathrm{~K}_{0}\right)$ a 30,94 $\left(\mathrm{N}_{3} \mathrm{~K}_{3}\right)$. A análise de regressão (Tabela 4) indica 
que houve efeito significativo apenas para componente linear da dose de nitrogênio, com o rendimento crescendo com a dose de N. Em cultivo de melão tipo amarelo, Coelho et al. (2001) verificaram efeito semelhante tanto para o N como para o K, estudando doses de até $180 \mathrm{~kg} \mathrm{ha}^{-1}$ de $\mathrm{Ne} 340 \mathrm{~kg} \mathrm{ha}^{-1} \mathrm{de}$ $\mathrm{K}_{2} \mathrm{O}$ em solo com teor de $\mathrm{K}$ no solo de $0,16 \mathrm{cmol}_{\mathrm{c}} \mathrm{kg}^{-1} \mathrm{em}$ cultivo de melão do tipo amarelo. No presente trabalho o teor de 0,18 $\mathrm{cmol}_{\mathrm{c}} \mathrm{kg}^{-1}$ de $\mathrm{K}$ presente no solo pode ser também uma provável justificativa na produtividade do melão obtida.

Tabela 4. Curva de acúmulo de nutrientes obtida no experimento de Bom Jesus para o melão Gália

\begin{tabular}{|c|c|c|c|c|c|}
\hline \multirow{2}{*}{ Tratamento } & \multicolumn{2}{|c|}{$\mathrm{N}$ na planta $\left(\mathrm{kg} \mathrm{ha}^{-1}\right)$} & \multicolumn{2}{|c|}{ K na planta $\left(\mathrm{kg} \mathrm{ha}^{-1}\right)$} & \multirow{2}{*}{$\begin{array}{r}\text { Produção } \\
\left(\mathrm{kg} \mathrm{ha}^{-1}\right)\end{array}$} \\
\hline & Obtido & Simulado & Obtido & Simulado & \\
\hline $\mathrm{N}_{1} \mathrm{~K}_{1}$ & 46,82 & 55,76 & 89,26 & 133,20 & 23,41 \\
\hline $\mathrm{N}_{2} \mathrm{~K}_{2}$ & 81,76 & 77,58 & 140,84 & 150,14 & 27,11 \\
\hline $\mathrm{N}_{3} \mathrm{~K}_{3}$ & 118,45 & 100,15 & 164,79 & 167,67 & 30,94 \\
\hline $\mathrm{N}_{2} \mathrm{~K}_{0}$ & 51,91 & 69,15 & 92,21 & 143,59 & 25,68 \\
\hline $\mathrm{N}_{0} \mathrm{~K}_{2}$ & 55,99 & 55,17 & 146,12 & 132,74 & 23,31 \\
\hline $\mathrm{N}_{0} \mathrm{~K}_{0}$ & 29,84 & 60,83 & 80,19 & 92,33 & 18,44 \\
\hline \multicolumn{6}{|c|}{ Prod $=20,3+0,0727^{* *} N\left(R^{2}=0,72\right)$} \\
\hline
\end{tabular}

** significativo a $1 \%$ pelo teste $t$ de Student

Constatou-se, através da análise de regressão, que a produtividade do melão sofre efeito significativo a $1 \%$ de probabilidade apenas para o efeito linear da dose de N (Tabela 4), enquanto para a melancia a produtividade sofre efeito significativo a $5 \%$ de probabilidade para o efeito quadrático das doses de N e K (Tabela 5). Para o melão pele de sapo, Dutra (2005) não verificaram efeito significativo das doses de N e K no número de frutos por planta. No caso deste tipo de melão normalmente a planta produz um fruto e mais importante é o seu tamanho, diferente do melão tipo gália, cujo rendimento

Tabela 5. Curva de acúmulo de nutrientes obtidos e simulados para a melancia M ickylee

\begin{tabular}{|c|c|c|c|c|c|}
\hline \multirow{2}{*}{ Tratamento } & \multicolumn{2}{|c|}{ N na planta ( $\mathrm{kg} \mathrm{ha}^{-1}$ ) } & \multicolumn{2}{|c|}{ K na planta $\left(\mathrm{kg} \mathrm{ha}^{-1}\right)$} & \multirow{2}{*}{$\begin{array}{r}\text { Produção } \\
\left(\mathrm{kg} \mathrm{ha}^{-1}\right)\end{array}$} \\
\hline & Obtido & Simulado & Obtido & Simulado & \\
\hline $\mathrm{N}_{0} \mathrm{~K}_{2}$ & 31,74 & 29,33 & 57,97 & 52,04 & 13.338 \\
\hline $\mathrm{N}_{2} \mathrm{~K}_{0}$ & 49,91 & 38,03 & 80,72 & 67,48 & 17.295 \\
\hline $\mathrm{N}_{1} \mathrm{~K}_{1}$ & 63,83 & 57,76 & 106,57 & 102,49 & 26.269 \\
\hline $\mathrm{N}_{1} \mathrm{~K}_{2}$ & 51,24 & 73,23 & 77,69 & 129,96 & 33.309 \\
\hline $\mathrm{N}_{1} \mathrm{~K}_{3}$ & 62,01 & 53,96 & 99,81 & 95,76 & 24.544 \\
\hline $\mathrm{N}_{2} \mathrm{~K}_{1}$ & 57,68 & 73,21 & 121,80 & 129,92 & 33.299 \\
\hline $\mathrm{N}_{2} \mathrm{~K}_{2}$ & 47,95 & 60,99 & 102,50 & 108,22 & 27.738 \\
\hline $\mathrm{N}_{2} \mathrm{~K}_{3}^{2}$ & 69,38 & 60,73 & 109,95 & 107,77 & 27.621 \\
\hline $\mathrm{N}_{3} \mathrm{~K}_{1}$ & 60,33 & 43,35 & 96,35 & 76,93 & 19.718 \\
\hline $\mathrm{N}_{3} \mathrm{~K}_{2}$ & 36,93 & 64,62 & 84,63 & 114,67 & 29.390 \\
\hline $\mathrm{N}_{3} \mathrm{~K}_{3}$ & 45,66 & 49,48 & 93,38 & 87,81 & 22.506 \\
\hline \multicolumn{6}{|c|}{ Prod $=-1561+\left(458^{*}\right) \mathrm{N}+\left(217^{*}\right) \mathrm{K}-\left(2,73^{*}\right) \mathrm{N}^{2}-\left(0,723^{*}\right) \mathrm{K}^{2}\left(\mathrm{R}^{2}=0,79\right)$} \\
\hline
\end{tabular}

deve estar muito mais associado ao número de frutos, haja vista que o mercado não requer frutos de grande calibre; já para a melancia, os dados do experimento de campo evidenciaram resultados semelhantes aos encontrados por Serafim (2006) para o efeito das doses de N e K.

Considerando o que postulam Teixeira et al. (2005), que citam $\mathrm{d}=1$ como perfeita concordância entre a eficiência do sistema em relação aos parâmetros testados e $\mathrm{d} \geq 0,7$ como valores aceitáveis, os valores do índice de concordância "d" evidenciam boa concordância para a equação de $\mathrm{K}$ da melancia e aceitáveis para as equações de $\mathrm{N}$ e $\mathrm{K}$ do melão e $\mathrm{N}$ da melancia (Tabela 6). Embora os valores de "d" sejam aceitáveis apenas para o melão, houve boa correlação entre os valores estimados e medidos nos experimentos de campo, o que pode ser explicado provavelmente pela absorção de luxo desses nutrientes, quando as doses foram muito elevadas. No caso da melancia, observaram-se valores elevados dos totais exportados de $\mathrm{N}$ e K (Tabela 5) revelando, neste caso, a ocorrência de uma interação maior entre os dados obtidos e os estimados para nutrição de $\mathrm{N}$ e $\mathrm{K}$ além de um ajuste maior do sistema nas situações locais.

\section{CONCLusões}

1. As melhores relações entre absorção total de nutrientes e rendimento, tanto para melancia quanto para o melão, foram produtividade (kg planta-1) x totais exportados ( g planta $\left.^{-1}\right)$.

2. O total exportado de nutrientes pelas plantas de melancia e melão em função da produtividade, é afetado pela densidade de plantio.

3. O modelo obtido a partir dos dados presentes na literatura estima, com relativa precisão, a necessidade nutricional líquida da melancia e do melão.

\section{LITERATURA CITADA}

Bar-Yosef, B. Advances in fertigation. Advances in Agronomy, v.65, p.1-77, 1999.

Coelho, E. F.; Sousa, V. F.; Souza V. A. B. Efeito de níveis de N e $\mathrm{K}$ aplicados por gotejamento na cultura do meloeiro (Cucumis melo L.) em solo arenoso. Ciência e Agrotecnologia, v.25, p.23-30, 2001.

Dutra, I. Produtividade e qualidade de frutos de melão pele de sapo em função de diferentes níveis de irrigação e adubações nitrogenada e potássica. Botucatu: UNESP, 2005. 99p. Tese Doutorado

Tabela 6. Valores do índice de concordância ("d") determinados para ascurvas médias que relacionam as quantidades exportadas dosnutrientes (g planta $\left.{ }^{-1}\right) X$ produtividade $\left(k_{\text {g planta }}^{-1}\right)$ para N e K e seus respectivos coeficientes de determinação $\left(R^{2}\right)$

\begin{tabular}{|c|c|c|c|c|}
\hline \multirow{2}{*}{$\begin{array}{c}\text { Cultura estudada } \\
\text { Nutriente }\end{array}$} & \multicolumn{2}{|c|}{ Melancia } & \multicolumn{2}{|c|}{ Melão } \\
\hline & $\mathbf{N}$ & K & $\mathbf{N}$ & $\mathbf{K}$ \\
\hline Índice "d" & 0,88 & 0,97 & 0,86 & 0,74 \\
\hline Coeficiente de determinação $\left(R^{2}\right)$ & 0,72 & 0,72 & 0,97 & 0,73 \\
\hline Equações que definiram o modelo & $y=1,202 x+0,8979$ & $y=1,934 x+3,0964$ & $y=5,8952 x-8,2243$ & $y=4,5784 x+2,6016$ \\
\hline
\end{tabular}


Faria, C. M. B.; Pereira, J. R.; Possideo, E. L. Adubação orgânica e mineral na cultura do melão em um vertissolo do submédio São Francisco. Pesquisa Agropecuária Brasileira, v.29, p.191-197, 1994.

Gärdenäs, A. I.; Hopmans, J. W.; Hanson, B. R.; Simunek, J. Two-dimensional modeling of nitrate leaching for various fertigation scenarios under micro-irrigation. Agricultural Water Management, v.74, p.219-242. 2005.

Grangeiro, L.C.; Cecílio Filho, A.B. Acúmulo e exportação de macronutrientes pela melancia sem sementes, híbrido Nova. Horticultura Brasileira, v.21, Suplemento 2, 2003. CD Rom

Grangeiro, L. C.; Cecílio Filho, A. B. Exportação de nutrientes pelos frutos de melancia em função de épocas de cultivo, fontes e doses de potássio. Horticultura Brasileira, v.22, p.740-743, 2004.

Grangeiro L. C.; Mendes, M. A. S.; Negreiros, M. Z. Acúmulo e exportação de nutrientes pela cultivar de melancia Mickylee. Caatinga, v.18, p.73-81. 2005.

Gurgel, M. T.; Uyeda, C. A.; Gheyi, H. R.; Oliveira, F. H. T.; Fernandes, P. D.; Silva, F. V. Crescimento de meloeiro sob estresse salino e doses de potássio. Revista Brasileira de Engenharia Agrícola e Ambiental, v.14, p.3-10, 2010.

Holanda Filho, R. S. F.; Sousa, V. F.; Azevedo, B. M.; Alcantara, R. M. C. M.; Ribeiro, V. Q.; Eloi, W. M. Efeitos da fertirrigação de $\mathrm{N}$ e K2O na absorção de macronutrientes pela gravioleira. Revista Brasileira de Engenharia Agrícola e Ambiental, v.10, p.43-49, 2006.

Kano, C.; Carmello, Q. A. C.; Cardoso, S. S.; Frizzone, J. A. Acúmulo de nutrientes pelo meloeiro rendilhado cultivado em ambiente protegido. Semina: Ciências Agrárias, v.31, p.1155-1164, 2010.

Kanwar, J. S.; Sekhon, G. S. Nutrient management for sustainable intensive agriculture. Fertiliser News, v.43, p.33-40, 1998

Lima, A. A. Adubação com NPK, via fertirrigação na cultura do meloeiro (Cucumis melo L.). Fortaleza, 2001. 53p. Dissertação Mestrado

Medeiros, J. F; Duarte, S. R.; Fernandes, P. D.; Dias, N. da S.; Gheyi, H. R. Crescimento e acúmulo de N, P e K pelo meloeiro irrigado com água salina. Horticultura Brasileira, v.26, p.452457, 2008.

Montag, U. J. Fertigation in Israel. Barcelona-Espanha: IFA Agricultural Conference on Managing Plant Nutrition, 1999, 24p.

Monteiro, R. O. C.; Colares, D. S; Costa, R. N. T.; Leão, M. C. S.; Aguiar, J. V. Função de resposta do meloeiro a diferentes lâminas de irrigação e doses de nitrogênio. Horticultura Brasileira, v.24, p.455-459, 2006.

Novais, R. F.; Smyth, T. J. Fósforo em solo e planta em condições tropicais. Viçosa: UFV, 1999. 399p.
Oliveira, F. H. T.; Novais, R. F.; Alvarez, V. H. V.; Cantarutti, R. B. Desenvolvimento de um sistema para recomendação de adubação para a cultura da bananeira. Revista Brasileira de Ciência do Solo, v.29, p.131-143, 2005.

Papadopoulos, I. Fertirrigação: Situação atual e perspectivas para o futuro. in: Folegatti, M.V. (coord.). Fertirrigação: Citrus, flores, hortaliças. Guaiba Agropecuária, 1999. 460p.

Paula, J. A. A. Desenvolvimento e verificação de sistema computacional para cálculo de adubação/fertirrigação em melão e melancia. Mossoró: UFERSA, 2007. 87p. Dissertação Mestrado

Pereira, A. B.; Villa Nova, N. A.; Alfaro, A. T. Necessidades hídricas de citros e macieiras a partir da área foliar e da energia solar. Revista Brasileira de Fruticultura, v.31, p.671679, 2009.

Pinto, J. M.; Soares, J. M.; Choudhury, E. N.; Pereira, J. R. Adubação via água de irrigação na cultura do melão. Pesquisa Agropecuária Brasileira, v.28, p.1263-1268, 1993.

Ribeiro Júnior, J. I. Análises estatísticas no SAEG. Viçosa: Folha de Viçosa, 2001. 301p.

Santos, F. C.; Neves, J. C. L.; Novais, R. F.; Alvarez, V. H. V.; Sediyama, C. S. Modelagem da recomendação de corretivos e fertilizantes para a cultura da soja. Revista Brasileira de Ciência do Solo, v.39, p.1661-1674, 2008.

Serafim, E. C. S. Modificações microclimáticas e acúmulo de nutrientes em melancia cultivada com proteção de agrotêxtil. Mossoró: UFERSA, 2006. 75p. Dissertação Mestrado

Silva, F. C. Manual de análises químicas de solos, plantas e fertilizantes. 1. ed. Rio de Janeiro: Embrapa solos. 1999. 370p.

Silva Júnior, M. J. Acúmulo de matéria seca e absorção de nutrientes pelo meloeiro "pele-de-sapo". Revista Brasileira de Engenharia Agrícola e Ambiental, v. 10, p.364-368, 2006.

Soares, J. M.; Brito, L. T. L.; Costa, N. D.; Maciel, J. L.; Faria, C. M. B. Efeito de fertilizantes nitrogenados na produtividade de melão. Pesquisa Agropecuária Brasileira, v.34, p.11391143, 1999.

Tedesco, M. J.; Gianello, C.; Bissani, C. A.; Bohnen, H.; Volkweiss, S. J. Análises de solo, plantas e outros materiais. 2. ed. Porto Alegre: UFRGS, 1995. 174p.

Teixeira, C. F. A.; Moraes, S. O.; Simonete, M. A. Desempenho do tensiômetro, TDR e sonda de nêutrons na determinação da umidade e condutividade hidráulica do solo. Revista Brasileira de Ciência do Solo, v.29, p.161-168, 2005.

Temóteo, A. da S.; Medeiros, J. F. de; Dutra, I.; Oliveira, F. de A. de. Crescimento e acúmulo de nitrogênio e potássio pelo melão pele de sapo fertirrigado. Irriga, v.15, p.275-281, 2010.

Vivancos, A. D. Fertirrigacion. Madri: Mundi - Prensa, 1999. $233 \mathrm{p}$. 